\title{
Performance of TETRA and GSM $\backslash$ GPRS as a Platform for Broadband Internet and Multimedia Evolution
}

\author{
M. I. Dababneh*1 and E. S. Al-Khawaldeh \\ $1^{*}$ Department of Electrical Engineering, Alisra University, Jordan \\ Received 19 Apr 2006; accepted 30 September 2006

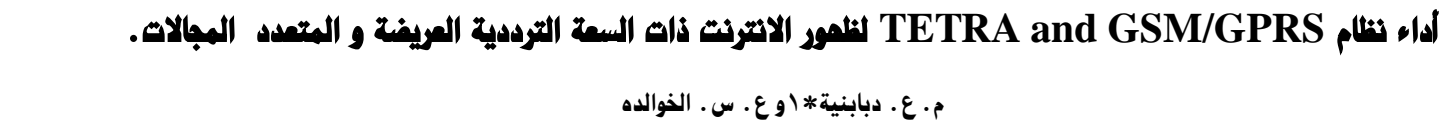 \\ الفلاصة: في هذه الورقة ، تم عرض طريقة جديدة ومبتكره لقارنة نظام TETRA ومنافسيه مبينين الكفاءات النسبية كما وتم عرض التطبيقات وامكانية الذدمات الجدية،

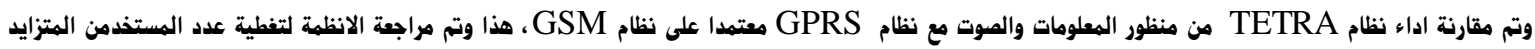 \\ باقصر وقت همكن وبالمحافظة على اعلى كفاءة ترددية همكنة. قياس لداء بث المعلومات يوضح ان نظام TETRA يملك وقت تجاوب اسع للتوصيل بالمقارنة هع ذلك لنظام

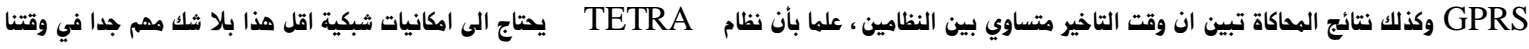 \\ الحاضر وخاصة ان هناك تزايد كبير على الاتصالات الخلوية واللاسلكية، بالاضافة الى ان الترددات ذات مصدر مددود وعلية يبب استغلال التردد بأفضل طريقة همكنة لنتككن من \\ تفطيتة الطلب البتزايدة على انظمة الاتصالات الخلوية.
}

الهزودات الهنتاهية: وقت تاسيس البكالبة ، العطاء، TETRA, GSM, GPRS

\begin{abstract}
In this paper, a novel approach for the comparison between TETRA system and other contenders is presented showing the relative potentials, thence applications and possible new services. TETRA Voice plus Data (V+D) and GPRSbased-GSM performance are also compared for defined multimedia transmission to revise the capability of both systems to afford multimedia applications to large number of subscribers with the shortest time possible while maintaining the optimal channel utilization. Performance measurements for data transmission illustrate that TETRA exhibits faster time response for connection setup time than the GPRS $\backslash$ GSM applications can provide. Simulation results also confirm that TETRA can support larger number of Mobile Stations (MSs) than the GSMIGPRS for the same time delay period, yet TETRA requires minimal network resources in comparison with GSM $\backslash$ GPRS for the same supported service. Indeed this is important in the present time where the demand on wireless communication is increasing dramatically and this what stimulates the concept of this research. Also, the hypothesis that certain communications systems utilizes spectrum efficiency more effectively than others and the fact that radio spectrum is a scarce recourse have further encourages the start of this research.
\end{abstract}

Keywords: TETRA, GSM, GPRS, Call setup-time, Throughput

\section{Introduction}

During the last decade, several standards for Mobile telecommunications have been developed. The most common is the Global System for Mobile Communications (GSM), standardized by the European Telecommunications Standards Institute (ETSI), as a cellular communications system operational in many countries around the globe. As GSM depends mainly on Integrated Digital Services Network (ISDN) standard (Rahnema, M., 1993), it is a successful platform for voice calls and data messages. Despite the exceptional features of GSM, it could not be considered as a typical stand for Public safety and Military applications requirements (Tetra Touch, Nokia Tetra Customer Newsletter, 2002;

*Corresponding author’s e-mail: m.dababneh@surrey.ac.uk
Heikonen, K. et al. 2002). Thus, a new brother for GSM is the trunked mobile radio system that is being implemented in Europe and is known as, Terrestrial Trunked Radio (TETRA), it is also developed by (ETSI) providing pure digital information technology for the transmission of speech and data. TETRA has been chosen as the platform for the operation of nationwide trunked radio networks in Europe; thus offering efficient and flexible multimedia communication services from both Private Mobile Radio (PMR) and Public-Access Mobile Radio (PAMR) users (ETSI, Terrestrial Trunked Radio, 2005).

As the cellular mobile communications market experiences hot competition, it is essential to compare the performance of the competing system types designed for data transmission such as the General Packet Radio System (GPRS) stemming from GSM and others with TETRA to pin point exactly the real usage and applications of the 
various systems depending on the associated characteristics, and to explore further services that were not originally planned for these systems.

Therefore, the aim of this paper is to study TETRA performance under the operating mode of $\mathrm{V}+\mathrm{D}$. Thus, comparing it with the GSM $\backslash$ GPRS network regarding the time delay and spectrum channel utilization needed to access the data by the MSs. It is essential however; that the network operators supply the subscribers with the required data under demand while maintaining the optimal channel utilization and maximum possible data rate (throughput).

This paper starts in Section 2 with an outline of TETRA together with an overview of the GSM $\backslash$ GPRS systems. TETRA network simulation analysis is then presented in Section 3 followed in Section 4 by performance evaluation of the GSM $\backslash$ GPRS and complete description of the system. Section 5 describes the relative performance between TETRA and GPRS networks. Finally, the conclusion section together with a set of recommendations concludes the paper.

\section{Overview of TETRA $(\mathrm{V}+\mathrm{D})$ and GSM/ GPRS Systems}

This section introduces the main features for TETRA $(\mathrm{V}+\mathrm{D})$ and GSM $\backslash$ GPRS systems. More information about both systems' protocols, signaling and architecture are beyond the subject of this paper and can be obtained from (Rahnema, M., 1993; Tetra Touch, Nokia Tetra Customer Newsletter, 2002; ETSI, Terrestrial Trunked Radio, 2005), and (Bettstetter, C, et al. 1999).

\subsection{Overview of TETRA $(V+D)$ System}

TETRA is a fully digital cellular Private Mobile Radio (PMR) architecture designed for reliable multimedia applications. The main reason to offer the new standard is that the need to provide a standardized network base for Public Safety and Security (PSS) that requires a network capable of support the following five main features detailed in (Tetra Touch, Nokia Tetra Customer Newsletter, 2002; Heikonen, K. et al. 2002):

- Group communications,

- Average response time in fractions of a second for both individual and group calls,

- Seamless radio coverage throughout the whole served area,

- Uncomposed and well-recognized high voice quality,

- Secure and authenticated connections.

These above requirements are entirely covered by TETRA. However, two modes of operations are defined for TETRA, one of which is the $(\mathrm{V}+\mathrm{D})$ mode of operation that allows a unique combination of point-to-point communication, point-to-multipoint communication, mobile telephony, and mobile data services accessible from one radio service (ETSI, Terrestrial Trunked Radio, 2005).

TETRA assigns uplink and downlink traffic separate- ly. It assigns a user up to four time slots per Time Division Multiple Access (TDMA) frame. When each slot transmits at a rate of $7.2 \mathrm{kbps}$, the four time slots, when combined, increases the data rate to $28.8 \mathrm{kbps}$ in an operation known as slot stealing, bandwidth on demand, or multislot operation. This feature makes TETRA an efficient standard for mobile communications having a frequency spectrum efficiency equivalent to $6.25 \mathrm{kHz}$ per channel per user.

Thus, TETRA is considered as a typical platform for data and multimedia applications such as short data messages, status messages and Internet protocol for packet data services. TETRA also supports generic and tailored data applications such as command and control systems, automatic vehicle locations, database queries, reporting, Wireless Access Protocol (WAP) solutions, image transfer, and video transmission (Lammerts, E, et al. 1999 and Shearer, E.H.S., 1995).

\subsection{Overview of GPRS Based-GSM System}

A practical extension of GSM is satisfied since 2001 when the service of General Packet Radio System (GPRS) is implemented in Europe and soon around the globe. GPRS is a new service bearer for GSM that greatly improves and simplifies wireless access to packet data networks, e.g., to the Internet. It transfers user data packets in an efficient way between GSM mobile stations and external packet data networks. Packets can be directly routed from the GPRS MSs to packet switched networks. Networks based on the Internet Protocol (IP) and X.25 networks are supported in GPRS (Bettstetter, C, et al. 1999 ).

As an improvement over GSM, GPRS supports multislot operation, in a similar way used in TETRA, such that each time slot allows data to be transmitted at a rate of 9.05 to $21.8 \mathrm{kbps}$ and up to $171.2 \mathrm{kbps}$ for the total 8 TDMA slots. As in TETRA, GPRS uplink and downlink traffics are allocated separately to provide higher efficiency. Under MSs request for transmission, GPRS cell allocates physical channels to support GPRS users called Packet Data Channels (PDCH)s where each PDCH contains multi logical channels for traffic and signaling (Bettstetter, C, et al. 1999).

\section{Performance Evaluation of TETRA}

\subsection{Performance Criteria and Simulation Model}

\subsubsection{Call Setup Time}

For PMR subscribers, one of the most important performance criteria is the call setup time. It is essential that a subscriber can request calls and sendldownload data as fast as possible. This depends critically on the call setup procedure used to access a channel. The frame timing for the call setup procedure considered in TETRA is shown in Fig. 1, which shows the call procedure for two mobile stations; MS A calling MS B via the Base station. The call setup time is defined as the time between the initial access 
request (U-SETUP message) sent by MS A and the allocation of traffic channel resources (D-CONNECT and DCONNECT ACKNOWLEDGE messages).

air interface air interface

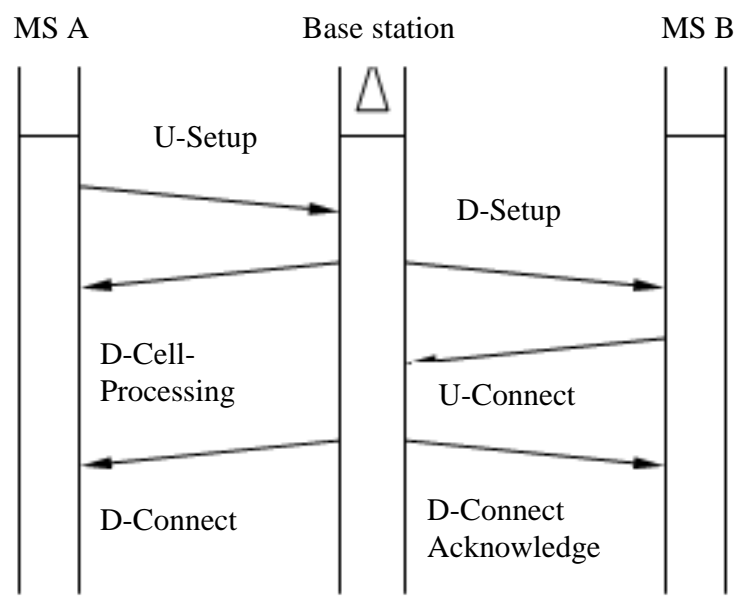

Figure 1. Frame timing of call setup time for TETRA system for point-point calls

However, this call procedure is used for individual (Point-to-Point) calls. The procedure is different in group (Multipoint) call communications where no acknowledgement message is required from MS B. Thus, the setup this time can be defined as the time elapsed between the sending of the (U-SETUP message) and the transmission of the (D-SETUP message).

In both procedures, if the system is loaded, MSs may try several attempts to gain access to the network. This will increase the delay such that the actual delay will equal the sum of the call setup time defined above and the waiting time for the MS to gain access to the traffic channel.

\subsubsection{Traffic Throughput and Traffic Load Performance Criterias}

Another important parameter to be considered in the analysis is the traffic throughput. It is defined for control channel during random access as the average number of successful call setups per frame; it is fully simulated in this analysis. The Throughput is affected by the Traffic Load in the network.

The Traffic Load is defined as the number of rejected calls per TDMA frame until $95 \%$ of the total call requests are succeeded. In other words, the offered traffic is an indication of the number of MSs requesting access to the network resources. As the number of MSs access the network increases, the traffic load is increased. Thus, it is expected that call setup time to be enlarged by the increased traffic load. This will become more apparent in Section 3.2.

The aim of the simulation study carried out in this section is to analyze the performance of TETRA network for call setup time delay and the throughput for both individual and group communications. The simulation of TETRA system protocols however, is performed with the following constraints in place:
- TETRA operates under $(\mathrm{V}+\mathrm{D})$ mode of operation

- 2500 MSs are uniformly distributed throughout the cell

- control channel activity is captured over 10,000 frames

- $\quad$ one TDMA frame duration $=56.67 \mathrm{~ms}$

- call generation is modeled as Poison process

- given the distance between a mobile and the base station, the path loss is calculated as the sum of the average path loss (Hata model) and a 1-path Rayleigh fading channel

- the transmission of each message is affected by noise and interference from other mobiles in contention. The statistical behaviour of this interference is assumed similar to additive white Gaussian noise

- $\quad$ power control has not been applied

- control channel capacity is reserved to support call set-up signaling

- it is assumed that sufficient traffic channels are available to support the offered traffic

- ALOHA access protocol is applied for uplink and downlink access with a back off time of 3 frames, an access window size of 6 , and the maximum number of transmission attempts (if failed) is 5 both uplink and downlink access.

\subsection{Analysis and Results of TETRA Performance}

A computer simulation is performed based on the simulation parameters above for both individual (Point to Point) and Group (Multipoint) calls. Figure 2 shows the Complementary Distribution Function (CDF) of percent call success against the Setup time for Point-to-Point calls under different traffic load conditions.

The statistical nature of Fig. 2 shows that to reach $95 \%$ of call success, an average of 35 TDMA frames (2 seconds) are required for the 0.1 traffic condition (13 success calls). This value increases to reach 140 TDMA frames (8 seconds) for 0.5 offered traffic (126 success calls). This yields that faster success call rate builds-up when the network exhibits less traffic load.

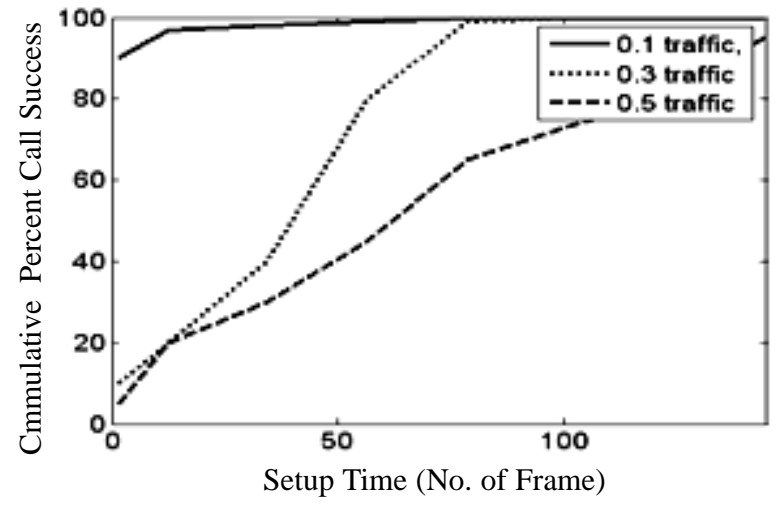

Figure 2. Set-up time for point-to-point calls 
It is clear that, in the case of Group (Multipoint) calls, however, the situation is better. The setup call procedure, discussed in Section 3.1, for Multipoint communications drops the number of messages required to establish a call and this in turn cuts down on loading the control channels hence leaving more capacity for initial random access.

Therefore, it is interesting for this research to compare the performance between the Point-to-Point and the Multipoint call procedures by considering Fig. 3. The results presented in Fig. 3 shows that, for the multipoint call procedure setup time measurements, to reach only 95\% cumulative, only less than 2 frames are required for the 0.1 traffic ( 9 success calls) and around 15 frames for 0.5 traffic load (250 success calls). That is there is a drop in setup time for the multipoint call procedure by a large percentage reaching $96 \%$ for the same traffic load.

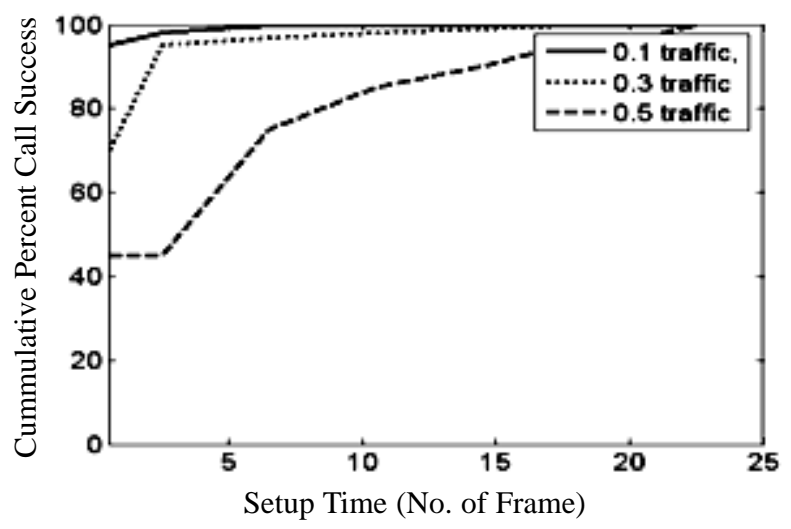

Figure 3. Set-up time for multipoint calls

Accordingly, much faster call setup times and enhanced TDMA frames utilization are achieved leaving the door open for more MSs to be served more willingly than the situation of Point-Point calls. For this purpose, the Multipoint call procedure is more suitable for group (multipoint) communications applications supported by TETRA.

The effect of the offered traffic load on the throughput is graphically depicted in Fig. 4 for Point-to-Point and Multipoint calls. In the case of Point-to-Point calls, it is evident that the throughput increases from 1 to 15 for increasing traffic condition from 0.1 to 0.5 . For the same

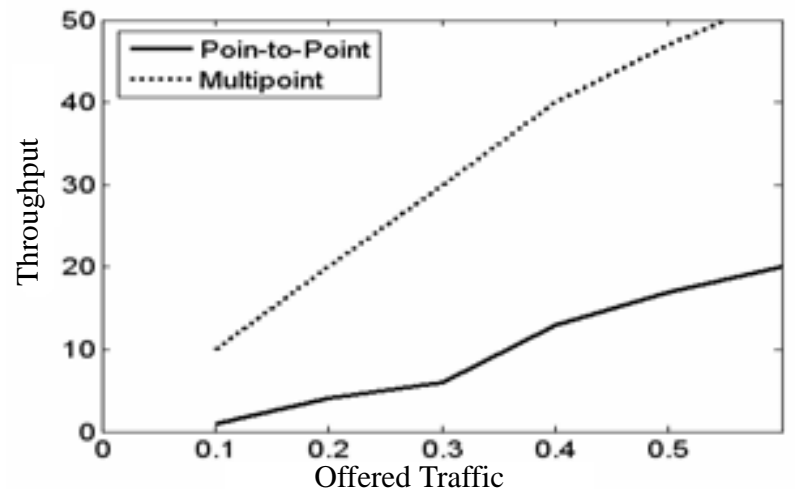

Figure 4. Throughput for point-to-point and multipoint calls traffic load, then the throughput increases from 10 to 47 in the case of Multipoint calls.

As a result, traffic throughput is clearly enhanced in the situation of Multipoint calls. Nevertheless, the throughput performance is degraded for an increasing traffic load for Point-to-Point and Multipoint call procedures. A summary of the above is shown in Table 1. It is obvious from Table 1 that the multipoint call procedure decreases the setup time required to reach 95\% success call rate.

Taking the 0.5 traffic load condition as a case of study, for the Point-to-Point call procedure, TETRA network rejects 126 MSs from 2506 MSs within 140 TDMA frames giving a throughput of 17 success call per frame. On the other hand, the multipoint call procedure rejects only 14 MSs from 264 MSs requesting access within the setup time of 5.3 TDMA frames, giving a throughput of 47 success calls per TDMA frame. Thus, for such condition of heavy traffic load, the throughput is enhanced by $176 \%$.

\section{Performance Evaluation of GPRS-based- GSM}

\subsection{Performance Criteria and Simulation Parameters}

GPRS is a service applied over GSM cellular mobile communications which has been established to transmit data in a rate of up to $171.2 \mathrm{kbit} / \mathrm{s}$. The simulation provides analysis for the GSM network based on GPRS service having two types of data applications; the e-mail and the WWW. The traffic in this case deals with two cases: one with pure WWW and e-mail, and other with mixed WWW and e-mail applications.

WWW is based on Hypertext Transfer Protocol (HTTP) layer which manages the transfer of the Hyper Text Markup Language (HTML) documents such as web pages (Shearer, E.H.S., 1997). Each webpage contains a number of objects with certain object size. E-mail depends mainly on the Simple Mail Transfer Protocol (SMTP) and the Post Office Protocol Version 3 (POP3). HTTP, SMTP and POP3 protocols are located in the application layer but the WWW and email traffic are carried through TCP $I P$ protocols (Bruce, A. Mah, 1997 and Loshin, P., 2000).

GPRS network performance can be measured using the following three main performance criteria's:

- Mean Application Response Time : defined as the difference between the time when a user is requesting a web page, or e -mail and the time when it is completely received.

- Downlink IP Throughput per user : is the number of IP bits transmitted in each TDMA frame.

- Packet Data Channel (PDCH) Utilization : is the number of MAC blocks utilized for MAC data and control data and control blocks normalized to the sum of data, control and idle blocks. 
Table 1. Relationship between the traffic load, number of MSs and setup time for point-to-point and multipoint call procedures

\begin{tabular}{|c|c|c|c|c|c|c|}
\hline & $\begin{array}{l}\text { Traffic } \\
\text { Load }\end{array}$ & Throughput & $\begin{array}{l}\text { Setup Time } \\
\text { to reach } \\
95 \% \text { call } \\
\text { success } \\
\text { [No. of } \\
\text { frames] }\end{array}$ & $\begin{array}{l}\text { No. of } \\
\text { succeeded } \\
\text { MSs = No. of } \\
\text { success calls }\end{array}$ & $\begin{array}{l}\text { No. of } \\
\text { rejected } \\
\text { MSs = No. of } \\
\text { rejected } \\
\text { calls }\end{array}$ & $\begin{array}{l}\text { Total No. of } \\
\text { MSs } \\
\text { requesting for } \\
\text { access = total } \\
\text { number of } \\
\text { calls }\end{array}$ \\
\hline \multirow{5}{*}{$\begin{array}{l}\text { Point-to- } \\
\text { Point }\end{array}$} & 0.1 & 1 & 35 & 35 & 2 & 37 \\
\hline & 0.2 & 4 & 60 & 240 & 13 & 253 \\
\hline & 0.3 & 6 & 88 & 528 & 28 & 556 \\
\hline & 0.4 & 13 & 118.3 & 1538 & 81 & 1619 \\
\hline & 0.5 & 17 & 140 & 2380 & 126 & 2506 \\
\hline \multirow{5}{*}{ Multipoint } & 0.1 & 10 & 0.88 & 9 & 1 & 10 \\
\hline & 0.2 & 20 & 1.77 & 36 & 2 & 38 \\
\hline & 0.3 & 30 & 2.65 & 80 & 5 & 85 \\
\hline & 0.4 & 40 & 3.53 & 142 & 8 & 150 \\
\hline & 0.5 & 47 & 5.3 & 250 & 14 & 264 \\
\hline
\end{tabular}

Table 2. WWW and e-mail traffic parameters

$\begin{array}{llll}\text { WWW Parameter } & \text { Distribution } & \text { Mean } & \text { Variance } \\ \text { Pages per session } & \text { Geometric } & 5.0 & 20.0 \\ \text { Intervals between pages [s] } & \text { negative exponential } & 12.0 & 144.0 \\ \text { Objects per page } & \text { Geometric } & 2.5 & 3.75 \\ \text { Object size [byte] } & \text { log2-Erlang-k (k=17) } & 3700 & 1.36 \times 10^{6} \\ & & & \\ & & & \\ \text { e-mail Parameter } & \text { Distribution } & \text { Mean } & \text { Variance } \\ \text { e-mail size (lower 80 \%) [byte] } & \text { log2-normal } & 1700 & 5.5 \times 10^{6} \\ \text { e-mail size (upper 20\%) [byte] } & \text { log2-normal } & 15700 & 62.9 \times 10^{9} \\ \text { Base quota [byte] } & \text { Constant } & 300 & 0\end{array}$

\subsubsection{GPRS Simulation Parameters}

A computer based simulation is implemented with parameters stated as below:

- Cluster size (7).

- $\quad$ Cell radius $=3000 \mathrm{~m}$.

- Velocity of MSs $=6 \mathrm{~km} / \mathrm{h}$.

- Acknowledged e-mail and WWW applications

- Multi-slot capability with one time slot for uplink and four time slots for downlink.

- Media Access Control (MAC) protocol is applied.

- The e-mail size is $(1700-15700)$ byte.

- The webpage size is (9250) byte.

- 4 Packet data Channels (PDCH) are considered.

- $\quad 30 \%$ of the traffic is assumed to be for e-mails and the rest is assumed to be for the WWW in the case of mixed traffic analysis.

The traffic load parameters of WWW and email are described in Table 2.

\subsubsection{GPRS Simulation Environment}

The Core of the simulator used in this paper is described in Fig. 5. The environment of the simulator is designed to comprise a MS model, a signal generator and a Base Station (BS) receives MS requests through the air interface (Um), a Serving GPRS Support Node (SGSN). All the components are integrated together to feed the statistical evaluation module.

\subsection{Analysis and Results of GPRS-based GSM Performance}

The performance of the GSM network under GPRS service is analyzed using computer simulation for both mixed and pure WWW and e-mail traffic. Figure 6 shows the results for the mean application response time for the case of pure WWWle-mail traffic. The response time increases from approximately two seconds at low traffic load to 20 seconds for the situation of heavy load. It is clear that WWW exhibits larger time period in compare with that of the e-mail time; as it contains more data. 


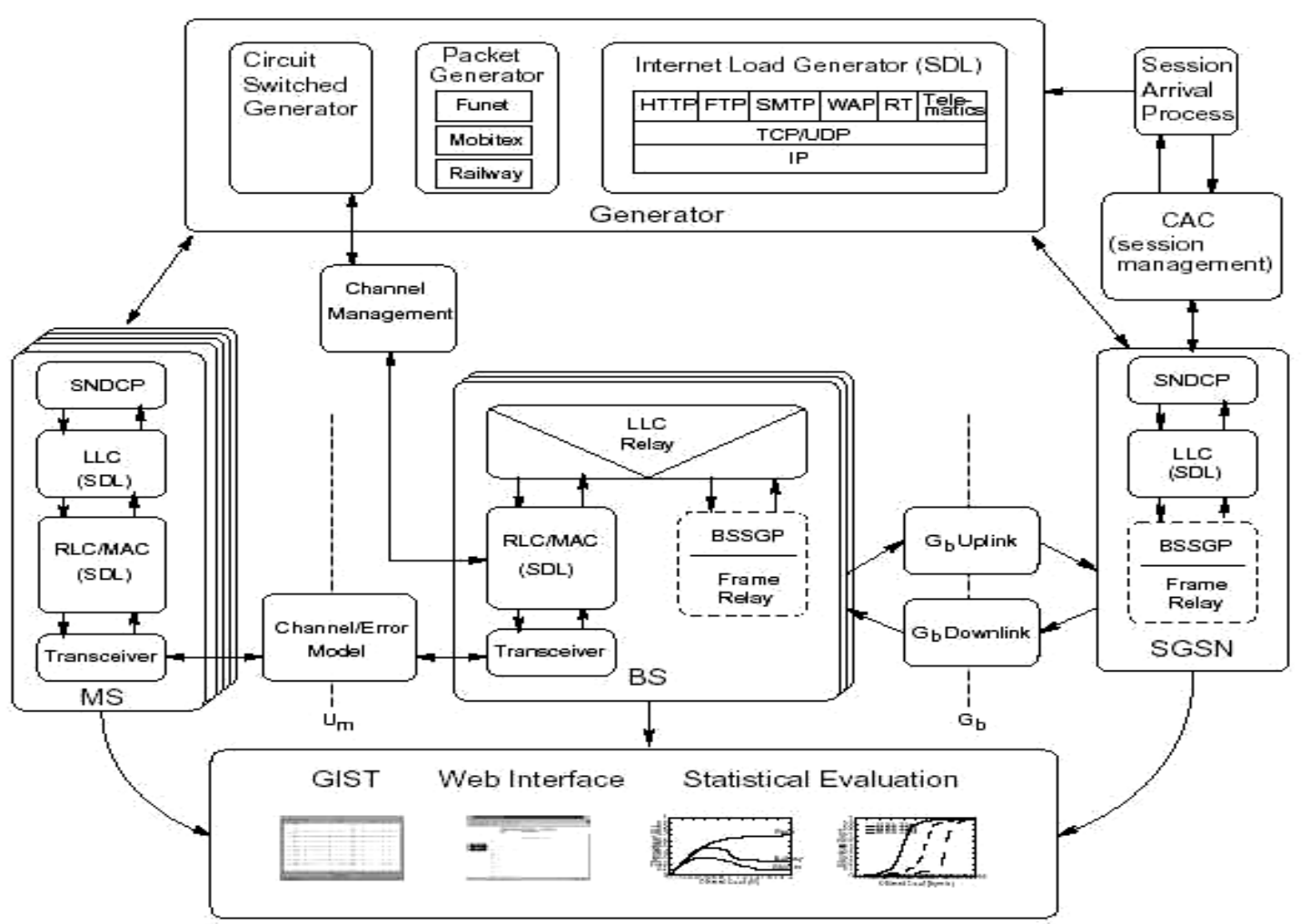

Figure 5. GRPS simulation environment

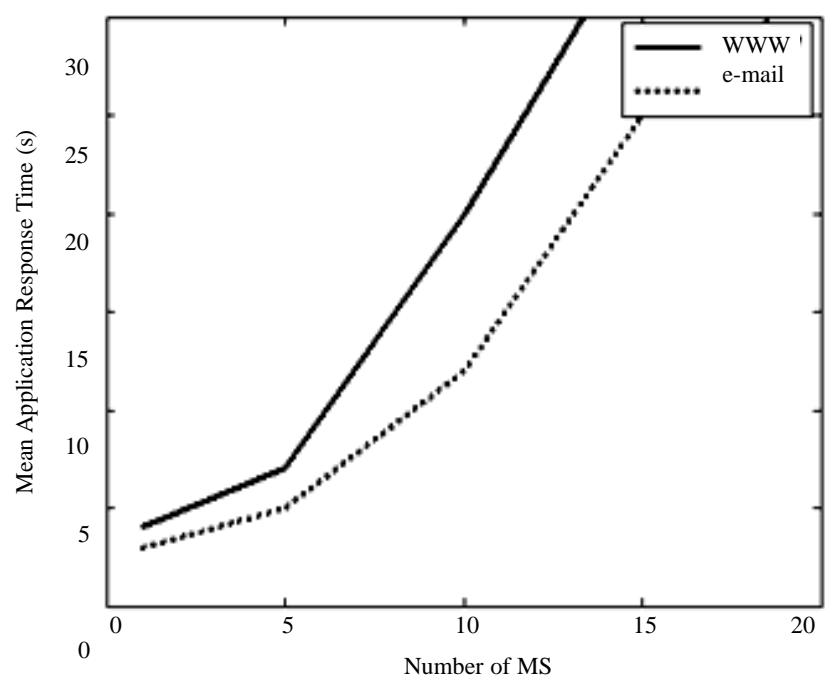

Figure 6. Mean application response time for pure WWWle-mail traffic

Figure 7 shows the application response time for the mixed WWWle-mail traffic. As in the case of pure traffic, the response time does increase as the number of active MSs increases, and in this case the time increases from 2 to 7 seconds compared with the case of pure traffic. As a result, the mixed traffic condition greatly speeds up the response time by $80 \%$ for heavy load conditions.

On the other hand, for the case of pure traffic, the reason for the large increase in the response time is apparent from the PDCH utilization measurements shown in Fig. 8 where in, approximately $100 \%$ utilization is reached when 15 MSs are competing to use the available PDCH channels.

In Fig. 9 however, the PDCH utilization seems to have

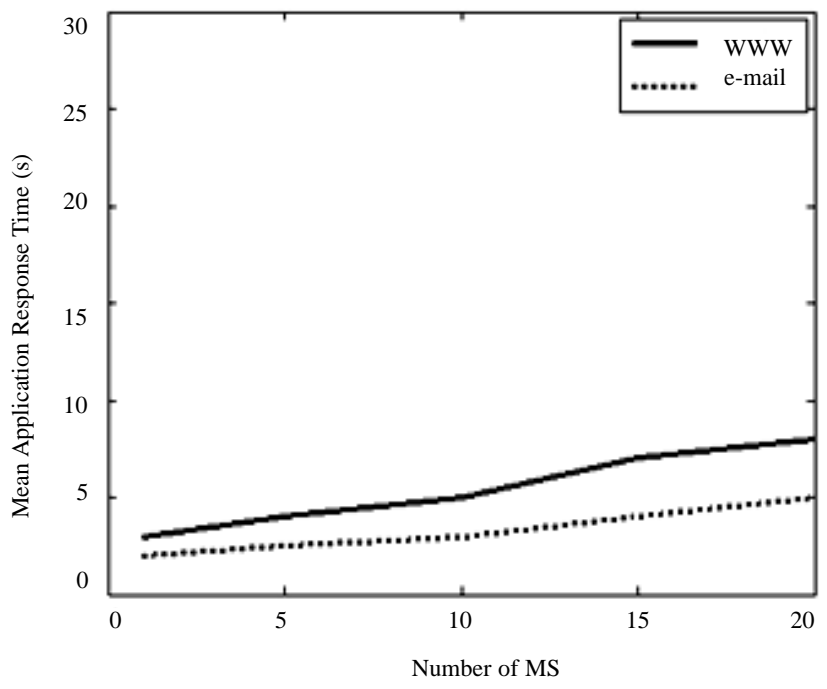

Figure 7. Mean application response time for mixed WWWle-mail traffic

linear relationship with the number of MSs. Therefore, instead of $100 \%$ utilization attained for pure traffic to serve $15 \mathrm{MSs}$, the mixed traffic decreases the PDCH utilization down to $70 \%$ for 20 MSs. Thus, the mixed traffic condition saves $30 \%$ of the available PDCHs and enables more MSs to access channel resources, thus resulting in better spectrum utilization.

Figure 10 shows graphically the measurements of the mean downlink IP throughput per user during transmission periods. It would be appropriate to discuss Fig. 10 and Fig. 8 of PDCH utilization together. The situation of 2 MSs illustrates that the network can offer high data rate (around 22kbitls) while utilizing $20 \%$ of the available PDCHs. As expected, the situation becomes poorer once 


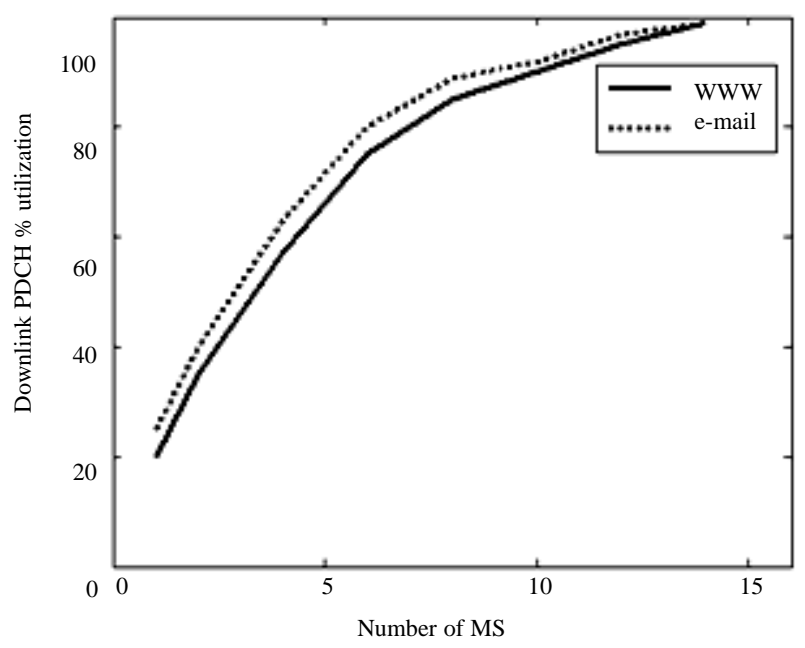

Figure 8. Mean downlink PDCH utilization for pure WWWle-mail traffic

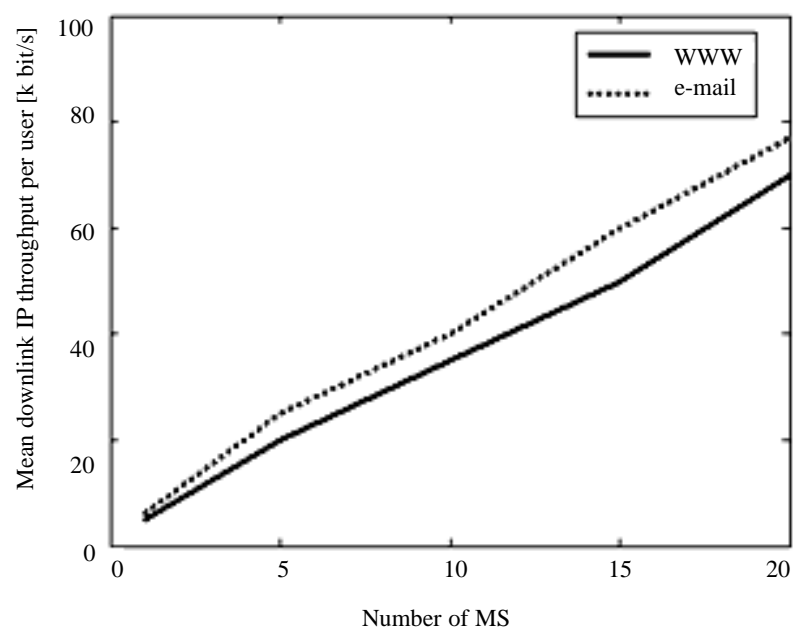

Figure 9. Mean downlink PDCH utilization for mixed WWWle-mail traffic

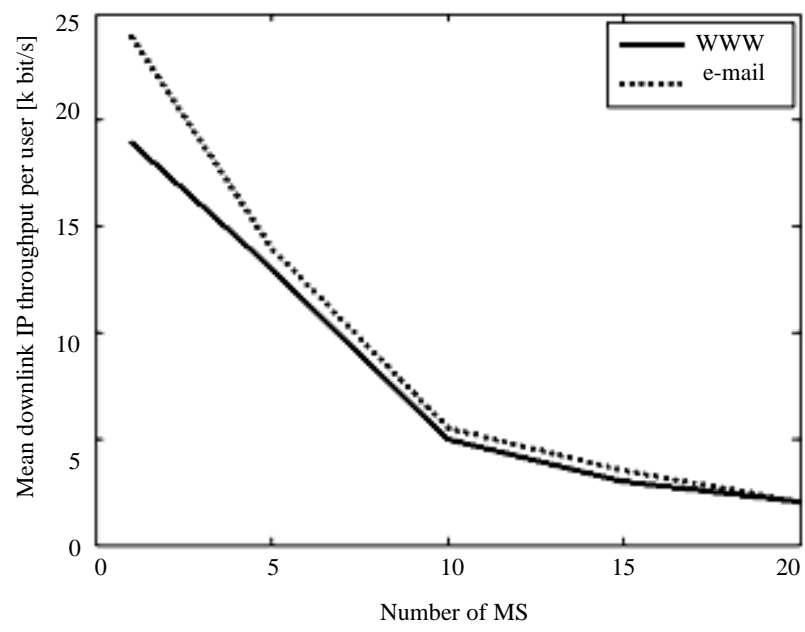

Figure 10. Mean downlink IP throughput for user per pure wwwle-mail traffic

the network is loaded by 15 MSs, in which the data rate is truncated down to be less than 5kbit/s and the PDCHs utilization is in excess of $80 \%$.
The throughput measurements seen in Fig. 11, indicates that its rate of decrease is slower than the case of pure traffic analysis shown in Fig. 10. Therefore, it is evident that the throughput decreases as the number of MSs increases, but this time from approximately 20kbit/s down to $10 \mathrm{kbit} / \mathrm{s}$ rather than 5kbit/s for pure traffic. Though, at heavy traffic load, the mixed traffic condition increases the downlink throughput data rate by approximately $100 \%$.

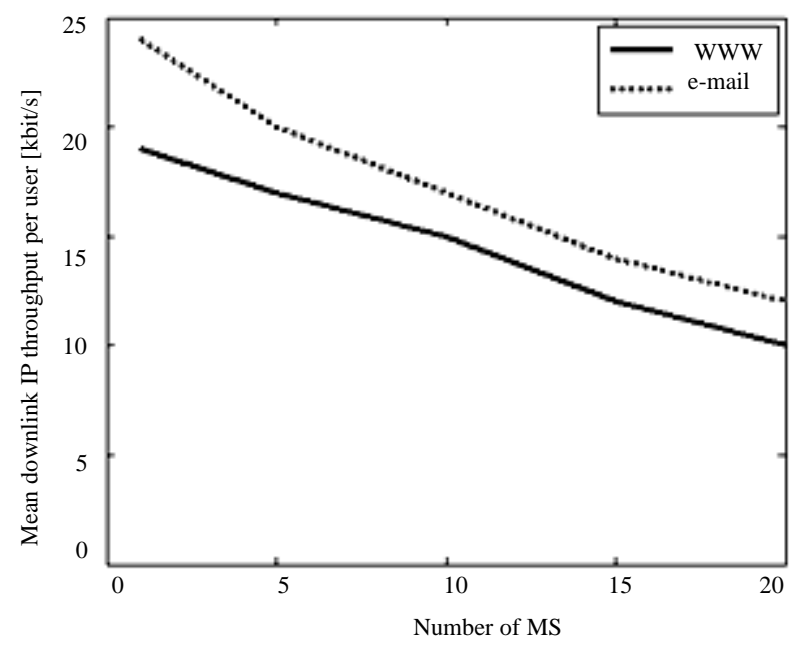

Figure 11. Mean downlink IP throughput for mixed WWWle-mail traffic

Furthermore, and for the case mixed traffic condition, the enhanced performance in the response time and throughput are due to the fact that better PDCH channel utilization is applicable for mixed traffic situation. This is obvious from Fig. 8 in comparison with Fig. 10.

Finally, comparing e-mail application with WWW application yields that the e-mails exhibits shorter application response time than the WWW. This comes from the fact that WWW web pages contain more data than the emails. This shows that WWW application has poorer PDCH utilization and throughput performance.

\section{Relative Performance between TETRA and GSM $\backslash$ GPRS}

An assessment for the study of TETRA and GSM/GPRS networks is provided in this section. Also, Simulation models for TETRA and GPRS networks under defined conditions have been implemented.

The relative performance criteria measurements for both network types are compared together. The setup time delay for the TETRA $(\mathrm{V}+\mathrm{D})$ network is compared to the WWWle-mail response time for the GPRS network. The relationship between the time delay measurements of both the throughput and the number of MSs is also discussed and fully analyzed.

\subsection{Setup Time in TETRA versus Application Response Time in GPRS}

A summary of the setup time delay in TETRA and the 
application response time in GPRS is provided in Table 3. Time delay measurements shown are chosen for different traffic load conditions. The e-mail traffic load condition is chosen as it consumes less response time than the WWW application. As a sample of measurements, to serve around 250 MSs, TETRA needs around (3.4 s) and (300 ms) for Point-to-Point and Multipoint call procedures respectively. On the other hand, the GPRS network serves 18 MSs during (30 s) and (5 s) for pure and mixed traffic respectively. It is apparent therefore that TETRA, in comparison to GPRS, serves much larger number of MSs for the same time duration and this results in shorter delay time.

The assessment of the two networks can not be completed until the throughput evaluation is revised with its relationship to the traffic load, time delay measurements as well as channel utilization.

\subsubsection{Throughput versus the number of MSs in TETRA and GPRS}

The definition of throughput performance criteria differs between TETRA and GPRS networks. In TETRA it is defined for control channel during random access as the average number of successful call setups per TDMA frame. In GPRS however, it is defined for the downlink IP packets; as the number of bits transmitted per TDMA frame per user.

In general, the throughput is an indication of the data rate of transmission or the speed of access and it is related to the number of MSs (traffic load) in the network.

To cope with the definition of the traffic throughput, defined in Section 3.1 for TETRA network; as the average number of MSs per total application response time is calculated in the GPRS network. procedures in TETRA support higher capacity for the offered traffic load. This is a manifest that TETRA strongly supports high capacity traffic and group calls suitable for PSS requirements.

The results of this comparison are shown in Figure 12 for TETRA network and in Fig. 13 for GPRS network. In Fig. 12, note that the throughput definition for TETRA network is measured per one second rather than per TDMA frame.

As a summary, Fig. 11 shows the measurements of the number of success calls per second, whereas Fig. 13 measures the number of completed applications per one second.

Therefore, it is clear that multipoint call procedure enhances the capacity for the random offered traffic compared to the Point-to-Point call procedure. In comparison to GPRS, It is evident that both call procedures in TETRA support higher capacity for the offered traffic load. This is a manifest that TETRA strongly supports high capacity traffic and group calls suitable for PSS requirements.

\section{Conclusions}

It is obvious from the simulation analysis that the GSM $\backslash G P R S$ network performance degrades as the num- ber of MSs is increased with limited number of $\mathrm{PDCH}$ channels. The mixed traffic condition shows superior performance relative to pure WWW and e-mail applications in the response time, IP throughput, and PDCH Utilization performance criterias. Thence, under heavy loaded network situations and mixed traffic condition the GSM $\backslash G P R S$ network saves approximately 30\% of the available PDCHs, increases the data rate by around $100 \%$ and deceases the response time by approximately $80 \%$.

The comparison between the results obtained from the GSM $\backslash$ GPRS network and the TETRA network states that TETRA performance is better in both: time delay and the number of mobile stations in the network. This leads to assert that TETRA strongly supports multimedia applications compared to GSM $\backslash$ GPRS network for the same time delay conditions.

In accordance with the lower number of control messages needed in the call procedure, it is found that TETRA call setup time for voice and data traffic in the case of group communications procedure is faster than in the case of individual calls. Furthermore, the throughput performance criterion for group call procedure enhances the network performance by $176 \%$ for the case of heavy traffic load of 0.5 , thence leave more traffic channels idle for random access.

Comparing the throughput measurements for both network types shows that, network performance degrades as traffic load in the network increases.

\section{References}

Bettstetter, C. Vögel, H. and Eberspacher, J., 1999, "Gsm Phase 2+ General Packet Radio Service Gprs: Architecture, Protocols, And Air Interface," IEEE Communications Surveys, http://www.comsoc.org/pubs/surveys, Vol. 2(3).

Bruce, A. Mah., 1997, "An Empirical Model of HTTP Network Traffic," Infocom, pp. 592, INFOCOM.

ETSI, 2005, "Terrestrial Trunked Radio (TETRA) Voice Plus Data (V+D). Part1, General Network Design; Final Draft," EN 300.3921 Vol..3.0.

Heikonen, K. and Saaristo, T., 2002, "You and Your TETRA RADIO, TETRA as a Tool for Public Safety," IT Press Edita Publishing Ltd.

Lammerts, E. Slump, C. H. et a., 1999, "Realization of a Mobile Data Application in TETRA," STW, 10 1901:038.

Loshin, P., 2000, "Essential Email Standards; RFCs and Protocols Made Practical," John Wiley \& Sons.

Rahnema, M., 1993, "Overview of the GSM System and Protocol Architecture," Communication Magazine, IEEE Vol. 31(4), pp. 92-100. ISSN 0163-6804.

Shearer, E.H.S., 1995, "TETRA - A Platform of Multimedia," Philips Telecom - PMR Cambridge.

"TETRA Touch, 2002, Nokia TETRA customer newsletter" www.nokia.com/tetra, Vol. 2. 